\title{
Fatty acids and aortic valve stenosis
}

\author{
Oscar Plunde, Magnus Bäck
}

Translational Cardiology, Center for Molecular Medicine, Department of Medicine Solna, Karolinska Institutet and Department of Cardiology, Karolinska University Hospital, Stockholm, Sweden

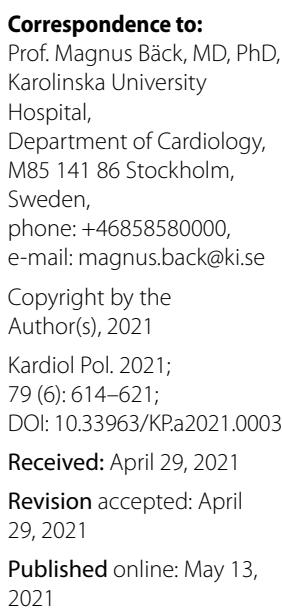

Correspondence to: Prof. Magnus Bäck, MD, PhD, Karolinska University Hospital, Department of Cardiology, M85 14186 Stockholm Sweden,

phone: +46858580000 e-mail: magnus.back@ki.se

Copyright by the

Author(s), 2021

Kardiol Pol. 2021:

79 (6): 614-621:

DOI: 10.33963/KP.a2021.0003

Received: April 29, 2021

Revision accepted: April 29, 2021

Published online: May 13, 2021

\author{
A B S T R A C T
}

Aortic valve stenosis (AVS), a valvulopathy that threatens life quality and longevity, in particular in an aging population. Yet no medical treatment is to date available emphasizing the need for more mechanistic insight into the disease to provide future treatment targets. Obesity and genetic variants within genes involved in lipid metabolisms and lipoprotein (a) have emerged as risk factors for AVS as these variants have significant genome-wide associations. The metamorphosis of the aortic valve to severe calcification involves lipid infiltration, inflammation, and oxidative stress which promotes further calcification in a viscous cycle in tandem with biomechanical factors that trigger further recruitment of inflammatory cells. The resolution of inflammation is an active and regulated process which therefore offers new possible targets. Fatty acids serve as substrate for many lipid mediators involved in the resolution of inflammation which may counterbalance the inflammation by promoting macrophage-mediated healing leading to a dampened inflammatory response. Recent data have put fatty acids in the spotlight as an important mechanism in the development of aortic valve disease. This review discusses possible mechanisms exerted by fatty acids in the context of AVS to facilitate future search for therapeutic targets. Key words: calcification, inflammation, lipid mediators, valvular heart disease

Kardiol Pol 2021; 79, 6: 614-621

\section{INTRODUCTION}

The most common indication for valve replacement is aortic valve stenosis [1] which affects over $10 \%$ of the population over 75 years of age [2]. Despite substantial advances, including reduced risks associated with surgical and catheter based techniques [3,4], potential advantages for alternatives to aortic valve intervention are stressing the need for a hitherto undiscovered effective medical treatment.

Several factors pave the way to severe AVS including modifiable lifestyle risk factors, genetic predisposition and congenital development defects e.g., bicuspid aortic valve. Risk factors associated with increased AVS incidence include diabetes [5], renal disease [6] and obesity [7]. The importance of the latter as a causal factor for AVS is illustrated by results from Mendelian Randomization (MR), in which body mass and fat mass indexes exhibit stronger associations with AVS compared with other cardiovascular diseases [8]. The genetic variants located in proximity to LPA and FADS1 have a genome-wide association with AVS $[9,10]$ and coronary artery disease [11] and acting through affecting levels of lipoprotein (a) (Lp(a)) and fatty acids, respectively. Interestingly, MR studies have shown a stronger association of these genetically determined risk factors with AVS compared with other cardiovascular diseases $[12,13]$. Each of these predisposing factors may influence the gradually fibrocalcific remodeling taking place locally in the aortic valve which ultimately may lead to a significant reduction in the aortic valve opening (Figure 1). The stenotic aortic valve has both hemodynamic and structural consequences to the left ventricle and if left untreated comes with a poor prognosis [14].

Given that obesity and specific lipids pathways are emerging as major risk factors for AVS, the present review aims to focus on fatty acids and their possible contribution to AVS development, by deciphering possible disease mechanisms affected by fatty acids and to evaluate the therapeutic potential within these pathways.

\section{INFLAMMATION}

Inflammation is a key component of the fibrocalcific remodeling in AVS and partakes throughout the continuum of the disease, driven by lipids. The commonly used inflammation marker C-reactive protein (CRP) is associated with AVS [15]. Calcified aortic valve tissue is a source for local production of cytokines e.g. interleukin-1 $\beta$ [16] and tumor necrosis 

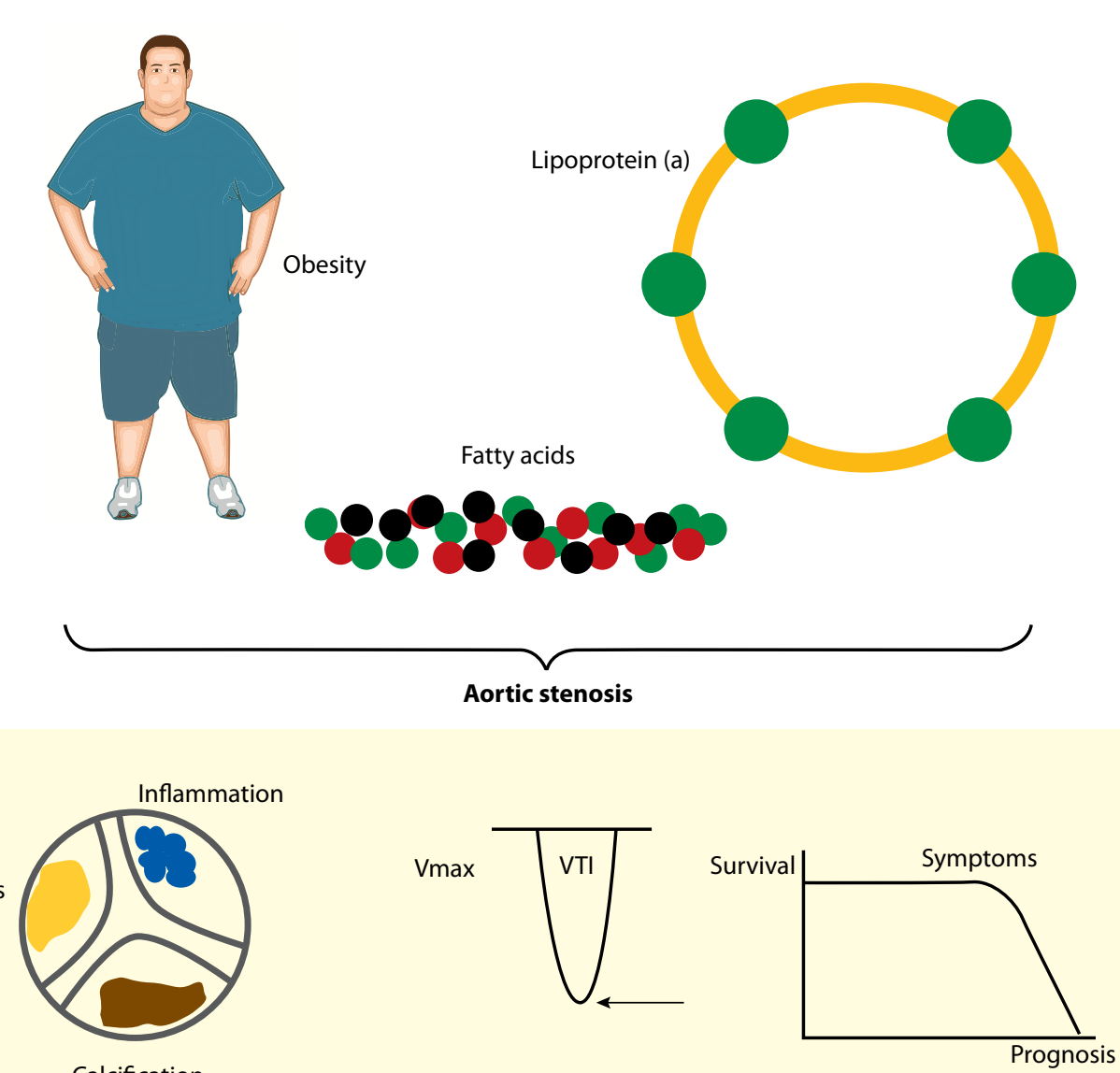

Calcification

Figure 1. Key components paving the way to aortic valve stenosis. Obesity, lipoprotein (a) (Lp[a]) and fatty acids are risk factors for aortic valve disease. These factors influence the pathophysiology locally in the aortic valve and partake in the progressive fibrocalcific remodelling by lipid and fatty acid alterations and affect severally inflammatory pathways ultimately leading to calcification. $L p(a)$ and fatty acid desaturase (FADS) are under the influence of genetic variants located in proximity to LPA and FADS1 which have shown genome-wide associations with aortic valve stenosis. The calcified aortic valve progressively turns immobile resulting in reduced valve orifice leading to increased transaortic peak velocity and pressure gradients, measured by echocardiography. When severe, only aortic valve replacement may ease the poor prognosis otherwise encountered ahead

factor-a (TNF-a) [17], as well as the proinflammatory lipid mediators prostaglandins [18] and leukotrienes [19, 20]. Increased mechanical stress potentiates the inflammation by endothelial cell activation which further propagates the influx of inflammatory cells [21] and lipids [22].

Inflammatory mediators give rise to a disturbance in the balance of synthesis and degradation of extracellular matrix (ECM) [23]. Malfunctioning ECM and matrix vesicles play a role in the initial stage of calcification acting as the foundation for dystrophic microcalcification [24, 25]. In response to inflammatory stimuli, valvular interstitial cells express osteogenic genes like bone morphogenic protein 2 (BMP2), Runx2, in addition to intracellular adhesion molecule 1 (ICAM-1), which also suggest a tight link between inflammation and calcification [26]. The coupling between inflammation and AVS also involves increased oxidative stress $[27,28]$. Finally, the initiation of the fibrocalcific process leads to increased mechanical stress, which acts to further propagate inflammatory pathways to drive the AVS disease forward.

Importantly, these processes are chronic, indicating a non-resolving aortic valve inflammation. The resolution of inflammation is a highly regulated process that balances the pro-inflammatory response by halting the influx of inflammatory cells, as well as promotes clearance of apoptotic polymorphonuclear leukocytes and immune cell efflux from sites of inflammation [29]. The balance between pro-inflammatory and pro-resolving mediators has gained great interest since it was found to be an actively regulated process and showed promise for future treatment targets [30]. In human aortic valves, the macrophage M2 phenotype is associated with the expression levels of proresolution mediating receptors [19] and pattern recognition receptors [31]. Fatty acids and in particular polyunsaturated fatty acids (PUFAs) are key components in this balance since they serve as substrates for lipid mediators exerting 
contradictory proinflammatory and pro-resolving actions potentially promoting progression and healing, respectively, of the AVS pathological circuits.

\section{FATTY ACIDS}

Fatty acids have been of interest for decades since Danish scientists discovered the beneficial cardiovascular effects of a diet rich in marine omega-3 fatty acids [32,33]. Greenland Inuits had lower mortality rates from myocardial infarction despite similar amount of total fat intake as Americans and Danes. The studies marked the beginning of the hypothesis that omega-3 PUFAs could be beneficial due to the favorable change in the ratio of downstream inflammatory and thrombotic mediators such as arachidonic acid (AA) and eicosapentaenoic acid (EPA). Following the release of $A A(C 20: 4 n 6)$ from the membrane by phospholipase $A 2, A A$ acts as a substrate for classical pro-inflammatory mediators. Catalyzed by the constitutively expressed cyclooxygenase (COX)- 1 and the inflammation induced COX-2, prostaglandins (PGs) and thromboxane A (TXA) are formed [34]. COX-derived products generally have pro-inflammatory and pro-thrombotic properties exemplified by TXA2 which facilitates clot formation. This is also supported by the effect of non-steroidal anti-inflammatory drugs (NSAIDs) [35]. AA also gives rise to leukotrienes via the 5-lipoxygenase (5-LO) pathway which promote inflammation in cardiovascular disease via leukotriene B4 (LTB4) among others [36]. Omega-3 EPA (C20:5n3), on the other hand, may act as a competing substrate for COX which results in the production of $\mathrm{PGI} 3$ with a similar beneficial profile as PGI2 produced with AA as substrate [37] and TXA3 which is less potent than TXA2 [38, 39]. In tandem, 5-LO catalyzed leukotriene production results in LTB5 which shows less activity compared to AA derived LTB4 and may therefore act as a competitive ligand for the common receptor resulting in a halted inflammatory response [40].

\section{CIRCULATING PUFAS AS BIOMARKERS}

High circulating levels of PUFAs measured as biomarkers have been associated with a higher chance of healthy aging [41]. Subjects within the highest quintile of docosapentaenoic acid (DPA), EPA and a composite of EPA, DPA and docosahexaenoic acid (DHA) concentration displayed significantly improved disease-free aging compared to the lowest quantile. This suggests that a relatively high concentration of omega- 3 fatty acids is needed to achieve beneficial effects. Interestingly, alpha-linolenic acid (ALA) was neutral for any association with unhealthy aging which suggests that the consumption of fish rich in EPA, DPA and DHA rather than the precursor is needed. This could also be a result of diverse individual genetics since the downstream modification of ALA is dependent on catalytic enzymes affected by polymorphisms within the gene encoding those enzymes. However, ALA measured in extracted phospholipids from plasma or total plasma was inversely correlated with fatal coronary heart disease as was DHA and EPA (a trend in total plasma) [42]. Importantly, a diet rich in seafood has been associated with other factors considered included in a healthy lifestyle [43] and the risk for residual confounding can therefore not be neglected. Also, shifting the balance of the omega-3/omega- 6 ratio was not interrogated and hence the results might have been impacted by lower omega- $6 \mathrm{PU}$ FAs rather than higher omega-3. However, a recent large meta-analysis suggested that higher circulating levels of the omega-6 PUFA linoleic acid (LA) associate with a lower risk of CVD and CVD mortality [44].

To our knowledge, associations of PUFAs in phospholipids extracted from plasma and AVS specifically only come from one genome-wide association study (GWAS) [10]. In pooled results from two previous studies, LA and ALA were inversely associated with aortic valve calcium (AVC), a predecessor of AVS. Furthermore, AA but not EPA was associated with AVC in addition to AVS.

\section{PUFAS IN THE AORTIC VALVE}

The pro-inflammatory and pro-resolution mediators can act locally making it reasonable to not only seek associations in the plasma but also locally in the valve. Indeed, lipids are present in aortic valves [45]. In calcified valves, higher amounts of oxidized lipids are found. The composition of PUFAs in aortic valves has been unknown until recently. Measured by gas chromatography as a percentage of total analyzed fatty acids, aortic valves contain high amounts of saturated palmitic acid (C16:0) and omega-9 oleic acid (C18:1n9). The most abundant PUFAs were linoleic acid (LA, C18:2n6), AA (C20:4n6) and DHA (C22:6n3) [46]. Interestingly, in a paired analysis comparing non-calcified and calcified parts within the same valve, the content of DHA was lower and gamma-linolenic acid (GLA) was higher in the calcified parts. This observation, which is summarized in Figure 3, strongly supports that aortic valve fatty acids are altered during AVS development.

\section{GENETIC DETERMINATION OF AORTIC VALVE PUFA METABOLISM}

Not only dietary intake determines the phospholipid composition but also genetic variants within genes encoding key enzymes in the metabolism of PUFAs. Of particular interest in the context of AVS are variants within the gene cluster in proximity to fatty acid desaturase (FADS) located on chromosome 11. FADS is a family of enzymes catalyzing the insertion of double bonds on PUFAs at specific positions. FADS1 encodes the delta-5-desaturase (D5D) activity enzyme that catalyzes the insertion of a double bond at carbon 5 yielding AA from dihomo- $\gamma$-linolenic acid (DGLA, C20:3n6) and EPA from eicosatetraenoic acid (ETA, C20:4n3). FADS2, located in the proximity of FADS1, encodes the delta-6-desaturase (D6D) activity enzyme that catalyzes the insertion of a double bond at carbon 6 yielding $\mathrm{Y}$-linolenic acid (GLA, C18:3n6) from linolenic acid (LA, C18:2n6) and stearidonic acid (SDA, C18:4n3) from a-linolenic acid (ALA, 


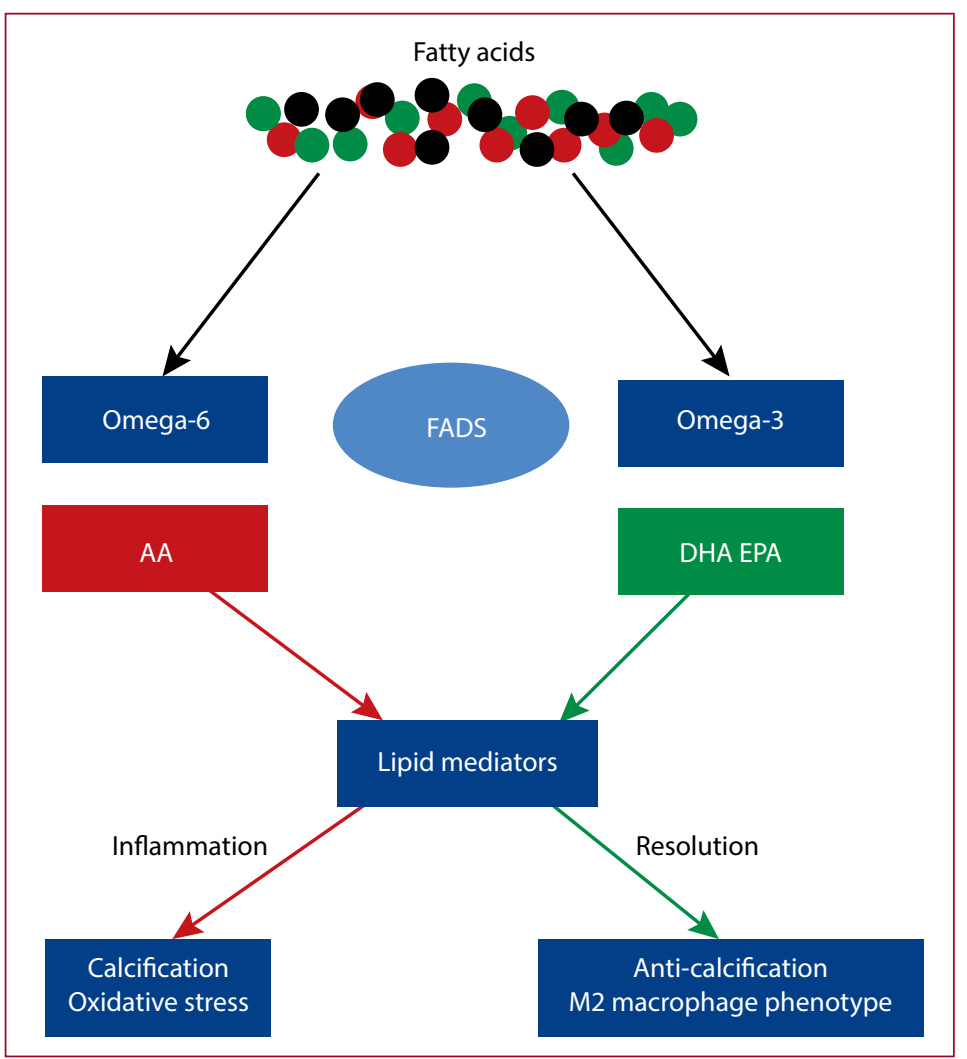

Figure 2. Metabolism of fatty acids by desaturase enzymes encoded by FADS. Rate-limiting enzymes encoded by fatty acid desaturase (FADS) $1 / 2$ are important in the production of polyunsaturated fatty acids that may be used for the production of lipid mediators. The omega- 6 fatty acid arachidonic acid (AA) gives rise to mediators prone to elicit a pro-inflammatory response which has been shown to increase the risk of calcification-related mechanisms in aortic valves. The omega-3 fatty acids docosahexaenoic acid (DHA) and eicosapentaenoic acid (EPA) on the other and act in favor of a pro-resolution response through downstream lipid mediators. The latter have a beneficial profile in the context of aortic valve calcification by halting the influx of inflammatory cells, clearing apoptotic cells and immune cell efflux

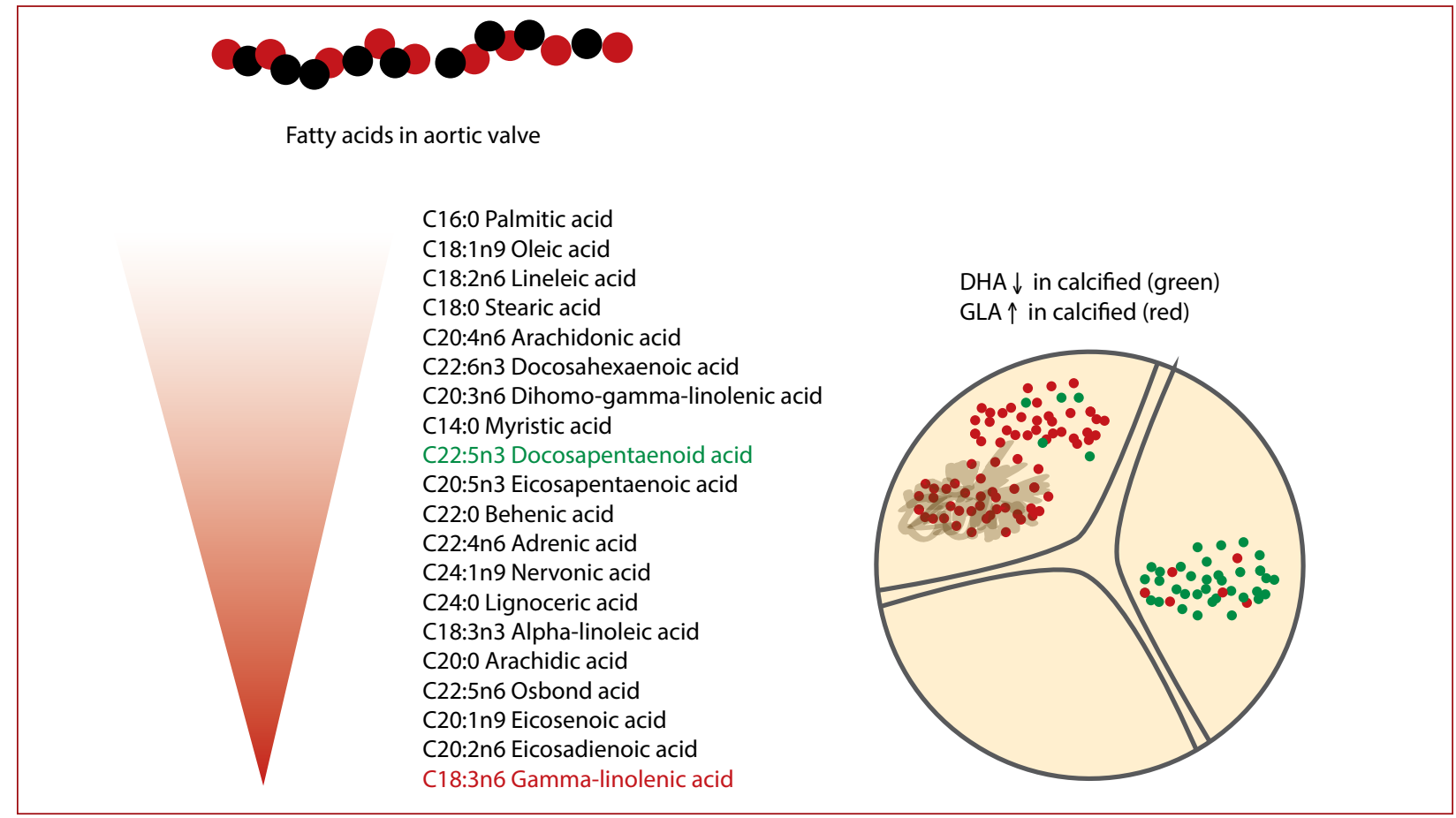

Figure 3. Fatty acids in the aortic valve and the implication in calcification. Levels of fatty acids in aortic valves (measured by gas chromatography as a percentage of total analyzed fatty acids) are presented with decreasing order. Aortic valves contain high amounts of saturated palmitic acid (C16:0) and omega-9 oleic acid (C18:1n9). The most abundant PUFAs are linoleic acid (LA, C18:2n6), AA (C20:4n6) and DHA (C22:6n3). Also, DHA is present in significantly higher levels in non-calcified tissue in contrast to gamma-linoleic acid with higher amounts in calcified tissue 
C18:3n3) [47]. FADS3 function remains to be elucidated [48]. These rate-limiting enzymes in the metabolism of PUFAs provide the strongest genetic influence on both PUFA blood concentrations [49] and D5D/D6D activity measured by-product to precursor ratio $[50,51]$. Single nucleotide polymorphism (SNP) rs174547 located in an intron within FADS1 demonstrated the most robust association with omega-6 [52] and with omega-3 PUFA ALA [49] in large GWAS studies. The minor C-allele was associated with lower D5D activity (low AA and high DGLA) and D6D activity (low GLA and high LA). SNP within the FADS cluster has further been implicated in several diseases including coronary artery disease [13, 53, 54], stroke [13], diabetes [55] and markers of inflammation in adipose tissue [56].

The minor FADS C-allele of the SNP rs 174575 is associated with an approximately $10 \%$ decreased risk of AVS in a GWAS [10] and similar results in a Mendelian randomization study of the minor FADS C-allele of rs174547 [13]. In the latter study, genetically determined (not including FADS1/2 variants) increased levels of $A A$ was associated with AVS. Importantly, since FADS1/2 variants have been associated with risk factors for AVS $[57,58]$, it might be difficult to fully account for pleiotropic effects even though rigorous efforts were made and the mechanisms behind these findings remain to be discovered. The genetic impact of FADS may be differently interpreted on a systemic and local level. Specifically, plasma levels of AA and the plasmatic AA to LA ratio were associated with both AVS and AVC [10], whereas neither EPA nor the EPA to ALA ratio exhibited significant associations with these phenotypes. In contrast, FADS-determined fatty acid levels in aortic valves showed the strongest associations with the omega-3 pathways [46].

\section{OMEGA-6 FATTY ACIDS IN AORTIC VALVE STENOSIS}

AA lipids are abundant in stenotic aortic valves [45]. Inhibiting the D5D in mice fed a western diet promotes amelioration of the atherosclerosis burden [59] which support the hypothesis that D5D-generated AA is indeed important for lipid-driven inflammation. Moreover, phospholipase A2 (PLA2) is responsible for making AA available for downstream metabolism. In aortic valves, PLA2 activity was associated with calcification and calcification-associated gene expression including BMP, osteopontin and alkaline phosphatase [60]. Also, COXs have been tightly linked to aortic valve calcification where upregulation of COX-2 leads to calcification [18, 60,61]. Furthermore, upregulation of 5-lipoxygenase which catalyze the first step in the cascade of leukotriene biosynthesis is upregulated in calcified compared to non-calcified regions in aortic valves [20]. The cysteinyl-leukotriene LTC4 is a potent stimulator of in vitro calcification of human valvular cells [20]. Additionally, hyperlipidemic mice with abolished FADS1-expression by anti-sense treatment had increased atherosclerotic burden in tandem with a balance in favor of the pro-inflammatory leukotrienes [62]. This emphasizes that lipid-derived pro-resolution mediators indeed impact AVS related mechanisms and are strongly influenced by the FADS1 genetic variant (Figure 2).

Although most AA-derived lipid mediators are proinflammatory, AA metabolism into lipoxins may balance the AA-induced inflammatory regulation. AA-derived lipoxins exert beneficial effects in murine atherosclerosis [63] and abdominal aortic aneurysms [64] what remains to be explored in AVS. The intricate balance between PUFAs is indeed complex and remains to be completely understood.

In the context of AVS, much is still unexplored but in the search for new therapeutic opportunities, one may link previous work on the mechanisms underlying AVS and PUFAs separately to drive hypothesis. In future work, the diverse effects of omega-6 PUFAs in AVS should not be neglected. In addition to AA, its upstream PUFA DGLA alleviates atherosclerosis and aortic calcification after supplementation in apolipoprotein E deficient mice [65]. This was accompanied by a decreased mRNA expression of adhesion molecules ICAM-1, VCAM-1 and NADPH oxidase subunits, which have all previously been shown to contribute to aortic valve inflammation and oxidative stress-driven calcification [21, 27, 66]. The observed beneficial effect could be the result of increased metabolism of prostaglandin E2 (PGE2) or DGLA itself and should be further evaluated. More recent in vitro studies have shown that DGLA decreases TNF-a independently of COX, suggesting that PGE2 does not account for the whole effect seen in in vivo studies [67]. Furthermore, DGLA dampens interferon- $\gamma$ induced adhesion molecules in macrophages in addition to increasing PGE1 concentration which itself partly attenuates IFN- $\gamma$ mediated inflammation [68]. Of importance, IFN- $\gamma$ has also been shown to partake in the aortic valve calcification [69].

The elongation product of AA Adrenic acid ( $\mathrm{AdA}$ ) shows beneficial pro-resolution effects in a murine peritonitis model [70]. Neutrophils may internalize AdA and when human primary neutrophils were stimulated, a significant reduction in LTB4 was found when AdA was administered. Interestingly, LTB4 is increased in calcified aortic valves $[19,71]$. While the role of neutrophils in AVS is poorly understood [72,73], macrophages, on the other hand, are key players in inflammation and aortic valve calcification. It is therefore important that AdA increases efferocytosis of apoptotic cells by macrophages [70] which could be of importance since apoptotic cells may serve as a nidus for calcification in aortic valves [74].

More studies are needed to fully appreciate the complete effects of the omega- 6 fatty acids in valvular inflammation, the above-mentioned studies enlighten the complexity of the often simplified balance between omega-6-derived lipid mediators and raise a novel notion that future therapeutic opportunities of fatty acids may arise also from specific omega-6 PUFAs. 


\section{OMEGA-3 FATTY ACIDS \\ IN AORTIC VALVE STENOSIS}

AVS patients with a higher omega-3 index locally in the explanted tricuspid aortic valve have echocardiographic signs of a slower AVS disease progression in the retrospective analysis [19]. A possible causality behind these observational human data is supported by the slower progression of murine aortic valve disease when genetically introducing endogenous omega-3 production. These mice have not only increased systemic but also increased valvular omega-3-levels, which reduced valve calcification but also improved the echocardiographic parameters similar to humans with high valvular omega-3 content.

The $\mathrm{D}$ - and E-series of resolvins (Rv) derived from $\mathrm{DHA}$ and EPA, respectively, have been identified in human aortic valves and raised the notion of these mediators as key omega-3-derived components in the resolution of valvular inflammation. In human aortic valves, both DHA-derived RvD3 and EPA-derived RvE1 are abundant in non-calcified but scarcely found in calcified tissue [19]. The exploration of valvular expression of the receptors for omega-3-derived lipid mediators points showed high levels of mRNA encoding the ChemR23 receptor for RvE1 in human aortic valve tissue. In support of the importance of this, ChemR23-/- hyperlipidemic mice display aggravated aortic valve disease compared with wild type mice [19]. Mechanistic exploration in mouse models and human aortic valves have supported direct valvular effects both as calcification inhibitors and by favoring a pro-resolving immune response by M2 macrophages. Taken together, this emerging evidence points towards the EPA-RvE1ChemR23 axis a crucial beneficial role of fatty acids in AVS, with possible therapeutic implications [75].

\section{THERAPEUTIC OPPORTUNITIES}

As AVS still stands without medical treatment, we encourage the scientific community to include studies on AVS outcome based on PUFA status and PUFA intervention. Future opportunities may arise from both purified PUFAs exemplified by the REDUCE-IT trial, in which the omega-3 fatty acid PUFA decreased all-cause mortality. In addition, future studies should further explore downstream lipid mediators, modifications of the receptors and enzymes in the fatty acid pathway may serve as therapeutic targets. To merely use diet as an intervention has a high risk of inconclusive data and will most likely be more sensitive to individual genetics. Therefore, considering that (i) aortic valves incorporate omega-3 PUFA (ii) high omega-3 fatty acids associate with slower progression av AVS (iii) EPA-derived RvE1 attenuates aortic valve disease in murine models and (iiii) high-dose EPA reduce all-cause mortality in high-risk individuals, provide a rationale for further clinical trials of high-dose EPA supplement as treatment for AVS.

\section{Article information}

Acknowledgements: The authors are supported by the Swedish Research Council (grant number 2019-01486), the Swedish Heart and Lung Foundation (grant number 20180571), and King Gustaf $V$ and Queen Victoria Freemason Foundation. OP is a fellow of the Clinical Scientist Training Programme (CSTP) at Karolinska Institutet.

Conflict of interest: None declared.

Open access: This article is available in open access under Creative Common Attribution-Non-Commercial-No Derivatives 4.0 International (CC BY-NC-ND 4.0) license, allowing to download articles and share them with others as long as they credit the authors and the publisher, but without permission to change them in any way or use them commercially. For commercial use, please contact the journal office at kardiologiapolska@ptkardio.pl.

How to cite: Plunde $\mathrm{O}$, Bäck M. Fatty acids and aortic valve stenosis. Kardiol Pol. 2021; 79(6): 614-621, doi: 10.33963/KP.a2021.0003.

\section{REFERENCES}

1. Nkomo VT, Gardin JM, Skelton TN, et al. Burden of valvular heart diseases: a population-based study. Lancet. 2006; 368(9540): 1005-1011, doi: 10.1016/S0140-6736(06)69208-8, indexed in Pubmed: 16980116.

2. Osnabrugge RLJ, Mylotte D, Head SJ, et al. Aortic stenosis in the elderly: disease prevalence and number of candidates for transcatheter aortic valve replacement: a meta-analysis and modeling study. J Am Coll Cardiol. 2013; 62(11): 1002-1012, doi: 10.1016/j.jacc.2013.05.015, indexed in Pubmed: 23727214.

3. Voigtländer $L$, Seiffert M. Expanding TAVI to low and intermediate risk patients. Front Cardiovasc Med. 2018; 5: 92, doi: 10.3389/fcvm.2018.00092, indexed in Pubmed: 30050909.

4. Baron SJ, Wang K, House JA, et al. Cost-effectiveness of transcatheter versus surgical aortic valve replacement in patients with severe aortic stenosis at intermediate risk. Circulation. 2019; 139(7): 877-888, doi: 10.1161/CIRCULATIONAHA.118.035236, indexed in Pubmed: 30586747.

5. Larsson SC, Wallin A, Håkansson N, et al. Type 1 and type 2 diabetes mellitus and incidence of seven cardiovascular diseases. Int J Cardiol. 2018; 262: 66-70, doi: 10.1016/j.ijcard.2018.03.099, indexed in Pubmed: 29605469.

6. Vavilis G, Bäck M, Occhino G, et al. Kidney dysfunction and the risk of developing aortic stenosis. J Am Coll Cardiol. 2019; 73(3): 305-314, doi: 10.1016/j.jacc.2018.10.068, indexed in Pubmed: 30678761.

7. Larsson SC, Wolk A, Håkansson N, et al. Overall and abdominal obesity and incident aortic valve stenosis: two prospective cohort studies. Eur Heart J. 2017; 38(28): 2192-2197, doi: 10.1093/eurheartj/ehx140, indexed in Pubmed: 28402538.

8. Larsson SC, Bäck M, Rees JMB, et al. Body mass index and body composition in relation to 14 cardiovascular conditions in UK Biobank: a Mendelian randomization study. Eur Heart J. 2020; 41(2): 221-226, doi: 10.1093/eurheartj/ehz388, indexed in Pubmed: 31195408.

9. Thanassoulis G, Campbell CY, Owens DS, et al. CHARGE Extracoronary Calcium Working Group. Genetic associations with valvular calcification and aortic stenosis. N Engl J Med. 2013; 368(6): 503-512, doi: 10.1056/NEJMoa1109034, indexed in Pubmed: 23388002.

10. Chen HY, Cairns BJ, Small AM, et al. Association of FADS1/2 locus variants and polyunsaturated fatty acids with aortic stenosis. JAMA Cardiol. 2020; 5(6): 694-702, doi: 10.1001/jamacardio.2020.0246, indexed in Pubmed: 32186652.

11. Clarke R, Peden JF, Hopewell JC, et al. PROCARDIS Consortium. Genetic variants associated with $\mathrm{Lp}(\mathrm{a})$ lipoprotein level and coronary disease. N Engl J Med. 2009; 361(26): 2518-2528, doi: 10.1056/NEJMoa0902604, indexed in Pubmed: 20032323.

12. Larsson SC, Gill D, Mason AM, et al. Lipoprotein(a) in Alzheimer, atherosclerotic, cerebrovascular, thrombotic, and valvular disease: mendelian randomization investigation. Circulation. 2020; 141(22): 1826-1828, doi: 10.1161/CIRCULATIONAHA.120.045826, indexed in Pubmed: 32479194.

13. Yuan S, Bäck M, Bruzelius $M$, et al. Plasma phospholipid fatty acids, and risk of 15 cardiovascular diseases: a mendelian randomisation study. 
Nutrients. 2019; 11(12): 3001, doi: 10.3390/nu11123001, indexed in Pubmed: 31817859.

14. Ross J, Braunwald E. Aortic stenosis. Circulation. 1968; 38(Suppl 1):61-67, doi: 10.1161/01.cir.38.1s5.v-61, indexed in Pubmed: 4894151.

15. Galante A, Pietroiusti A, Vellini $M$, et al. C-reactive protein is increased in patients with degenerative aortic valvular stenosis. J Am Coll Cardiol. 2001; 38(4): 1078-1082, doi: 10.1016/s0735-1097(01)01484-x, indexed in Pubmed: 11583885.

16. Kaden JJ, Dempfle CE, Grobholz R, et al. Interleukin-1 beta promotes matrix metalloproteinase expression and cell proliferation in calcific aortic valve stenosis. Atherosclerosis. 2003; 170(2): 205-211, doi: 10.1016/s00219150(03)00284-3, indexed in Pubmed: 14612199.

17. Leopold JA. Cellular mechanisms of aortic valve calcification. Circ Cardiovasc Interv. 2012; 5(4): 605-614, doi: 10.1161/CIRCINTERVENTIONS.112.971028, indexed in Pubmed: 22896576.

18. Wirrig EE, Gomez MV, Hinton RB, et al. COX2 inhibition reduces aortic valve calcification in vivo. Arterioscler Thromb Vasc Biol. 2015; 35(4): 938-947, doi: 10.1161/ATVBAHA.114.305159, indexed in Pubmed: 25722432.

19. Artiach $\mathrm{G}$, Carracedo $M$, Plunde $\mathrm{O}$, et al. Omega-3 polyunsaturated fatty acids decrease aortic valve disease through the Resolvin E1 and ChemR23 Axis. Circulation. 2020; 142(8): 776-789, doi: 10.1161/CIRCULATIONAHA.119.041868, indexed in Pubmed: 32506925.

20. Nagy E, Andersson DC, Caidahl K, et al. Upregulation of the 5-lipoxygenase pathway in human aortic valves correlates with severity of stenosis and leads to leukotriene-induced effects on valvular myofibroblasts. Circulation. 2011; 123(12): 1316-1325, doi: 10.1161/CIRCULATIONAHA.110.966846, indexed in Pubmed: 21403093.

21. Aikawa $E$, Nahrendorf $M$, Sosnovik $D$, et al. Multimodality molecular imaging identifies proteolytic and osteogenic activities in early aortic valve disease. Circulation. 2007; 115(3): 377-386, doi: 10.1161/CIRCULATIONAHA.106.654913, indexed in Pubmed: 17224478.

22. Olsson M, Thyberg J, Nilsson J. Presence of oxidized low density lipoprotein in nonrheumatic stenotic aortic valves. Arterioscler Thromb Vasc Biol. 1999; 19(5): 1218-1222, doi: 10.1161/01.atv.19.5.1218, indexed in Pubmed: 10323772.

23. Hutson HN, Marohl T, Anderson M, et al. Calcific aortic valve disease is associated with layer-specific alterations in collagen architecture. PLoS One. 2016; 11(9): e0163858, doi: 10.1371/journal.pone.0163858, indexed in Pubmed: 27685946.

24. New SEP, Aikawa E. Molecular imaging insights into early inflammatory stages of arterial and aortic valve calcification. Circ Res. 2011; 108(11): 1381-1391, doi: 10.1161/CIRCRESAHA.110.234146, indexed in Pubmed: 21617135.

25. Bäck M, Michel JB. From organic and inorganic phosphates to valvular and vascular calcifications. Cardiovasc Res. 2021 [Epub ahead of print], doi: 10.1093/cvr/cvab038, indexed in Pubmed: 33576771.

26. Bogdanova M, Kostina A, Zihlavnikova Enayati $K$, et al. Inflammation and mechanical stress stimulate osteogenic differentiation of human aortic valve interstitial cells. Front Physiol. 2018; 9: 1635, doi: 10.3389/fphys.2018.01635, indexed in Pubmed: 30524301.

27. Miller JD, Chu Yi, Brooks RM, et al. Dysregulation of antioxidant mechanisms contributes to increased oxidative stress in calcific aortic valvular stenosis in humans. J Am Coll Cardiol. 2008; 52(10): 843-850, doi: 10.1016/j.jacc.2008.05.043, indexed in Pubmed: 18755348.

28. Mercier N, Pawelzik SC, Pirault J, et al. Semicarbazide-sensitive amine oxidase increases in calcific aortic valve stenosis and contributes to valvular interstitial cell calcification. Oxid Med Cell Longev. 2020; 2020: 5197376, doi: 10.1155/2020/5197376, indexed in Pubmed: 32411328.

29. Bäck M, Yurdagul A, Tabas I, et al. Inflammation and its resolution in atherosclerosis: mediators and therapeutic opportunities. Nat Rev Cardiol. 2019; 16(7): 389-406, doi: 10.1038/s41569-019-0169-2, indexed in Pubmed: 30846875.

30. Serhan CN. Pro-resolving lipid mediators are leads for resolution physiology. Nature. 2014;510(7503): 92-101, doi: 10.1038/nature13479, indexed in Pubmed: 24899309.

31. Karadimou G, Plunde O, Pawelzik SC, et al. TLR7 expression is associated with M2 macrophage subset in calcific aortic valve stenosis. Cells. 2020; 9(7): 1710, doi: 10.3390/cells9071710, indexed in Pubmed: 32708790.
32. Bang HO, Dyerberg J, Hjøorne N. The composition of food consumed by Greenland Eskimos. Acta Med Scand. 1976; 200(1-2): 69-73, doi: 10.1111/j.0954-6820.1976.tb08198.x, indexed in Pubmed: 961471.

33. Dyerberg J, Bang HO, Stoffersen E, et al. Eicosapentaenoic acid and prevention of thrombosis and atherosclerosis? Lancet. 1978; 2(8081): 117-119, doi: 10.1016/s0140-6736(78)91505-2, indexed in Pubmed: 78322.

34. Cipollone F, Cicolini G, Bucci M. Cyclooxygenase and prostaglandin synthases in atherosclerosis: recent insights and future perspectives. Pharmacol Ther. 2008; 118(2): 161-180, doi: 10.1016/j.pharmthera.2008.01.002, indexed in Pubmed: 18420277.

35. Sala A, Proschak E, Steinhilber D, et al. Two-pronged approach to anti-inflammatory therapy through the modulation of the arachidonic acid cascade. Biochem Pharmacol. 2018; 158: 161-173, doi: 10.1016/j. bcp.2018.10.007, indexed in Pubmed: 30315753.

36. BäckM. Leukotriene signaling in atherosclerosis and ischemia. Cardiovasc Drugs Ther. 2009; 23(1):41-48, doi: 10.1007/s10557-008-6140-9, indexed in Pubmed: 18949546.

37. BäckM. Omega-3 fatty acids in atherosclerosis and coronary artery disease. Future Sci OA. 2017; 3(4):FSO236, doi: 10.4155/fsoa-2017-0067, indexed in Pubmed: 29134121.

38. Vane JR, Botting RM. Heart disease, aspirin, and fish oil. Circulation. 1991; 84(6): 2588-2590, doi: 10.1161/01.cir.84.6.2588, indexed in Pubmed: 1959207.

39. Force T, Milani R, Hibberd P, et al. Aspirin-induced decline in prostacyclin production in patients with coronary artery disease is due to decreased endoperoxide shift. Analysis of the effects of a combination of aspirin and $n-3$ fatty acids on the eicosanoid profile. Circulation. 1991; 84(6): 2286-2293, doi: 10.1161/01.cir.84.6.2286, indexed in Pubmed: 1959184.

40. Bäck M, Hansson GK. Omega-3 fatty acids, cardiovascular risk, and the resolution of inflammation. FASEB J. 2019; 33(2): 1536-1539, doi: 10.1096/f.201802445R, indexed in Pubmed: 30703872.

41. Lai HTM, de Oliveira Otto MC, Lemaitre RN, et al. Serial circulating omega 3 polyunsaturated fatty acids and healthy ageing among older adults in the Cardiovascular Health Study: prospective cohort study. BMJ. 2018; 363: k4067, doi: 10.1136/bmj.k4067, indexed in Pubmed: 30333104.

42. Del Gobbo LC, Imamura F, Aslibekyan S, et al. Cohorts for Heart and Aging Research in Genomic Epidemiology (CHARGE) Fatty Acids and Outcomes Research Consortium (FORCe). $\omega-3$ polyunsaturated fatty acid biomarkers and coronary heart disease: pooling project of 19 cohort studies. JAMA Intern Med. 2016; 176(8): 1155-1166, doi: 10.1001/jamainternmed.2016.2925, indexed in Pubmed: 27357102.

43. Wennberg M, Tornevi A, Johansson I, et al. Diet and lifestyle factors associated with fish consumption in men and women: a study of whether gender differences can result in gender-specific confounding. Nutr J.2012; 11: 101, doi: 10.1186/1475-2891-11-101, indexed in Pubmed: 23210480.

44. Marklund M, Wu JHY, Imamura F, et al. Cohorts for Heart and Aging Research in Genomic Epidemiology (CHARGE) Fatty Acids and Outcomes Research Consortium (FORCE). Biomarkers of Dietary Omega-6 Fatty Acids and Incident Cardiovascular Disease and Mortality. Circulation. 2019; 139(21): 2422-2436, doi: 10.1161/CIRCULATIONAHA.118.038908, indexed in Pubmed: 30971107.

45. Lehti S, Käkelä R, HörkköS, et al. Modified lipoprotein-derived lipid particles accumulate in human stenotic aortic valves. PLoS One. 2013; 8(6): e65810, doi: 10.1371/journal.pone.0065810, indexed in Pubmed: 23762432.

46. Plunde O, Larsson SC, Artiach G, et al. FADS1 (fatty acid desaturase 1) genotype associates with aortic valve FADS mRNA expression, fatty acid content and calcification. Circ Genom Precis Med. 2020; 13(3): e002710, doi: 10.1161/CIRCGEN.119.002710, indexed in Pubmed: 32397743.

47. Lee JM, Lee $H$, Kang $S$, et al. Fatty acid desaturases, polyunsaturated fatty acid regulation, and biotechnological advances. Nutrients. 2016; 8(1):23, doi: 10.3390/nu8010023, indexed in Pubmed: 26742061.

48. Blanchard $H$, Legrand $P$, Pédrono F. Fatty acid desaturase 3 (fads 3 ) is a singular member of the fads cluster. Biochimie. 2011; 93(1): 87-90, doi: 10.1016/j.biochi.2010.03.002, indexed in Pubmed: 20226833.

49. Lemaitre RN, Tanaka T, Tang W, et al. Genetic loci associated with plasma phospholipid n-3 fatty acids: a meta-analysis of genome-wide association studies from the CHARGE Consortium. PLoS Genet. 2011; 7(7): e1002193, doi: 10.1371/journal.pgen.1002193, indexed in Pubmed: 21829377. 
50. Marklund M, Morris AP, Mahajan A, et al. Genome-wide association studies of estimated fatty acid desaturase activity in serum and adipose tissue in elderly individuals: associations with insulin sensitivity. Nutrients. 2018; 10(11): 1791, doi: 10.3390/nu10111791, indexed in Pubmed: 30453627.

51. de Toro-Martín J, Guénard F, Rudkowska I, et al. A common variant in ARHGEF10 alters delta- 6 desaturase activity and influence susceptibility to hypertriglyceridemia.J Clin Lipidol. 2018; 12(2):311-320.e3, doi: 10.1016/j. jacl.2017.10.020, indexed in Pubmed: 29246731.

52. Guan W, Steffen BT, Lemaitre RN, et al. Genome-wide association study of plasma N6 polyunsaturated fatty acids within the cohorts for heart and aging research in genomic epidemiology consortium. Circ Cardiovasc Genet. 2014; 7(3): 321-331, doi: 10.1161/CIRCGENETICS.113.000208, indexed in Pubmed: 24823311.

53. Martinelli N, Girelli D, Malerba G, et al. FADS genotypes and desaturase activity estimated by the ratio of arachidonic acid to linoleic acid are associated with inflammation and coronary artery disease. Am JClin Nutr. 2008; 88(4): 941-949, doi: 10.1093/ajcn/88.4.941, indexed in Pubmed: 18842780.

54. Lu Y, Vaarhorst A, Merry AHH, et al. Markers of endogenous desaturase activity and risk of coronary heart disease in the CAREMA cohort study. PLoSOne. 2012; 7(7):e41681, doi:10.1371/journal.pone.0041681, indexed in Pubmed: 22911844.

55. Kröger J, Zietemann V, Enzenbach C, et al. Erythrocyte membrane phospholipid fatty acids, desaturase activity, and dietary fatty acids in relation to risk of type 2 diabetes in the European Prospective Investigation into Cancer and Nutrition (EPIC) — Potsdam Study. Am J Clin Nutr. 2011; 93(1): 127-142, doi: 10.3945/ajcn.110.005447, indexed in Pubmed: 20980488.

56. Vaittinen $\mathrm{M}$, Walle $\mathrm{P}$, Kuosmanen $\mathrm{E}$, et al. FADS2 genotype regulates delta- 6 desaturase activity and inflammation in human adipose tissue. J Lipid Res. 2016; 57(1): 56-65, doi: 10.1194/jlr.M059113, indexed in Pubmed: 26609056.

57. Ching YK, Chin YS, Appukutty M, et al. Interaction of dietary linoleic acid and a-Linolenic acids with rs174547 in gene on metabolic syndrome components among vegetarians. Nutrients. 2019; 11(7): 1686, doi: 10.3390/nu11071686, indexed in Pubmed: 31340443.

58. Yuan S, Larsson SC. Association of genetic variants related to plasma fatty acids with type 2 diabetes mellitus and glycaemic traits: a Mendelian randomisation study. Diabetologia. 2020; 63(1): 116-123, doi: 10.1007/s00125-019-05019-0, indexed in Pubmed: 31690987.

59. Takagahara S, Shinohara $\mathrm{H}$, Itokawa $\mathrm{S}$, et al. A novel orally available delta-5 desaturase inhibitor prevents atherosclerotic lesions accompanied by changes in fatty acid composition and eicosanoid production in knockout mice. J Pharmacol Exp Ther. 2019; 371(2): 290-298, doi: 10.1124/jpet.119.259846, indexed in Pubmed: 31488602

60. Suzuki K, Takahashi S, Watanabe K, et al. The expression of groups IIE and $\mathrm{V}$ phospholipase $\mathrm{A} 2$ is associated with an increased expression of osteogenic molecules in human calcified aortic valves. J Atheroscler Thromb. 2014; 21(12): 1308-1325, doi: 10.5551/jat.24273, indexed in Pubmed: 25132377.

61. Sakaue T, Hamaguchi M, Aono J, et al. Valve interstitial cell-specific cyclooxygenase-1 associated with calcification of aortic valves. Ann Thorac Surg. 2020; 110(1):40-49, doi: 10.1016/j.athoracsur.2019.09.085, indexed in Pubmed: 31760051.

62. Gromovsky AD, Schugar RC, Brown AL, et al. $\Delta-5$ fatty acid desaturase impacts metabolic disease by balancing proinflammatory and proresolving lipid mediators. Arterioscler Thromb Vasc Biol. 2018; 38(1): 218-231, doi: 10.1161/ATVBAHA.117.309660, indexed in Pubmed: 29074585.

63. Petri MH, Laguna-Fernandez A, Arnardottir $\mathrm{H}$, et al. Aspirin-triggered lipoxin A4 inhibits atherosclerosis progression in apolipoprotein E -/mice. Br J Pharmacol. 2017; 174(22): 4043-4054, doi: 10.1111/bph.13707, indexed in Pubmed: 28071789.

64. Petri MH, Thul S, Andonova T, et al. Resolution of inflammation through the lipoxin and ALX/FPR2 receptor pathway protects against abdominal aortic aneurysms. JACC Basic TransI Sci. 2018;3(6):719-727, doi: 10.1016/j. jacbts.2018.08.005, indexed in Pubmed: 30623131.

65. Takai $\mathrm{S}$, Jin D, Kawashima $\mathrm{H}$, et al. Anti-atherosclerotic effects of dihomo-gamma-linolenic acid in ApoE-deficient mice. J Atheroscler Thromb. 2009; 16(4): 480-489, doi: 10.5551/jat.no430, indexed in Pubmed: 19713674.

66. Liu $\mathrm{H}$, Wang $\mathrm{L}$, Pan $Y$, et al. Celastrol alleviates aortic valve calcification via inhibition of NADPH oxidase 2 in valvular interstitial cells. JACC Basic TransI Sci. 2020; 5(1): 35-49, doi: 10.1016/j.jacbts.2019.10.004, indexed in Pubmed: 32043019.

67. Dooper MM, van Riel B, Graus YMF, et al. Dihomo-gamma-linolenic acid inhibits tumour necrosis factor-alpha production by human leucocytes independently of cyclooxygenase activity. Immunology. 2003; 110(3): 348-357, doi: 10.1046/j.1365-2567.2003.01749.x, indexed in Pubmed: 14632663.

68. Gallagher H, Williams JO, Ferekidis N, et al. Dihomo-y-linolenic acid inhibits several key cellular processes associated with atherosclerosis. Biochim Biophys Acta Mol Basis Dis. 2019; 1865(9): 2538-2550, doi: 10.1016/j. bbadis.2019.06.011, indexed in Pubmed: 31202985.

69. Nagy E, Lei Y, Martínez-Martínez E, et al. Interferon- $\gamma$ released by activated CD8T lymphocytes impairs the calcium resorption potential of osteoclasts in calcified human aortic valves. Am JPathol. 2017; 187(6): 1413-1425, doi: 10.1016/j.ajpath.2017.02.012, indexed in Pubmed: 28431214

70. Brouwers $\mathrm{H}$, Jónasdóttir $\mathrm{HS}$, Kuipers $\mathrm{ME}$, et al. Anti-inflammatory and proresolving effects of the omega- 6 polyunsaturated fatty acid adrenic acid. J Immunol. 2020; 205(10): 2840-2849, doi: 10.4049/jimmunol.1801653, indexed in Pubmed: 33008950.

71. Kochtebane N, Passefort S, Choqueux C, et al. Release of leukotriene B4, transforming growth factor-beta1 and microparticles in relation to aortic valve calcification. J Heart Valve Dis. 2013; 22(6): 782-788, indexed in Pubmed: 24597398.

72. Li S, Xu L, Liu B. The neglected role of neutrophils in the severity of aortic valve stenosis. J Cardiovasc Pharmacol. 2019; 74(5): 367-368, doi: 10.1097/FJC.0000000000000737, indexed in Pubmed: 31517777.

73. Kopytek M, Kolasa-Trela R, Zabczyk M, et al. NETosis is associated with the severity of aortic stenosis: links with inflammation. Int J Cardiol. 2019; 286: 121-126, doi: 10.1016/j.ijcard.2019.03.047, indexed in Pubmed: 30952530.

74. Proudfoot D, Skepper JN, Hegyi L, et al. Apoptosis regulates human vascular calcification in vitro: evidence for initiation of vascular calcification by apoptotic bodies. Circ Res. 2000; 87(11): 1055-1062, doi: 10.1161/01. res.87.11.1055, indexed in Pubmed: 11090552.

75. Artiach G, BäckM. Omega-3 polyunsaturated fatty acids and the resolution of inflammation: novel therapeutic opportunities for aortic valve stenosis? Front Cell Dev Biol. 2020; 8: 584128, doi: 10.3389/fcell.2020.584128, indexed in Pubmed: 33304901. 